${ }^{1} 0$ Beser, ${ }^{2}$ E Celik, ${ }^{2} \mathrm{~S}$ Lacinel, ${ }^{1} \mathrm{~T}$ Erkan, ${ }^{1} \mathrm{~T}$ Kutlu, ${ }^{1} \mathrm{FC}$ Cokugras. ${ }^{1}$ Pediatric Gastroenterology, Hepatology and Nutrition; '2Dept. of Pediatrics, Istanbul University Cerrahpasa Medical Faculty, Istanbul, Turkey

Introduction Eosinophilic esophagitis(EOE) is a chronic esophageal inflammatory disease. Because signs and symptoms of the disease are similar to the gastroesophagial reflux (GER) disease many patients are treated as GERdisease.

Aim In this study we aim to evaluate 42 children with the diagnosis of EOE.

Methods Clinical and laboratuary databases of 42 patients who were followed-up.

Results $57 \%$ of patients was male, mean age 7.24 \pm 3.8 .IgE levels were high in 24 patients of the all patients. Skin prick test was applied to the patients who have high IgE levels however the factor that may cause allergies could be detected in $1 / 2$ of them. We applied gastroscopy to all patients and nodular appearance was seen in $90 \%$ of them. Biopsy specimens of all cases had at least 15 eosinophils infiltration. Food elimination was performed to 12 patients whose allergic factor was detected by skin pirck test. Antihistaminic treatement was applied to 12 cases and four of them had systemic steroid treatment. 8 cases were treated by montelukast and topical steroid treatment. Complaints of thirty eight patients who did not have systemic steroid treatment were relieved in the end of first month however four patients that had to use steroids relieved in the end of the second month.

Conclusion EOE is increasing everyday. Complaints and the findings are similar to much of GER disease however it should be considered first in patients whose complaints are going on desipite the GERD treatment. Treatment costs and the quality of life is high in terms of early response to treatment in pediatric patients which is recognizable form of the disease is gaining importance.

\section{MONITORING FAECAL CALPROTECTIN LEVELS IN THE FOLLOW UP OF DISEASE ACTIVITY AND TREATMENT OF COW'S MILK ALLERGY}

doi:10.1136/archdischild-2012-302724.0693

'S Sancak, ${ }^{2} \mathrm{OF}$ Beser, ${ }^{2} \mathrm{~T}$ Erkan, ${ }^{2} \mathrm{~T}$ Kutlu, ${ }^{2} \mathrm{FC}$ Cokugras. ${ }^{1}$ S. B. Zeynep Kamil Kadin ve Cocuk Hastalıkları E.A. Hastanesi; ${ }^{2}$ Pediatric Gastroenterology, Hepatology and Nutrition, Istanbul University Cerrahpasa Medical Faculty, Istanbul, Turkey

Aim Faecal Calprotectin is recommended as a noninvasive indicative marker of the extent of intestinal mucosal inflammation associated with inflammatory bowel disease and cow's milk allergy (CMA). We aim to demonstrate the usability of measuring faecal Calprotectin as a simple, noninvasive and reliable test in following up disease activity and pointing out relapses in patients with gastrointestinal symptoms associated with colorectal inflammation.

Methods Newly diagnosed 32patients were recruited into the CMA group while 19 healthy symptomless subjects were included into the control group.

Results Patients in the CMA group were shown to exhibit higher faecal Calprotectin levels than their controls. The levels were significantly lower in the CMA patients after diet modification than they were before diet modification. Patients in İgE mediated group fecal calprotectin level were found higher than control group, but there were no significant difference istatistically. Patients in nonİgE mediated group had istatistically higher fecal calprotectin levels than controls. Patients in non-İgE mediated group had higher level of faecal calprotectin than patients in IgE mediated group. After exclution diet, we observed significant regression of fecal calprotectin in both groups.

Conclusion It has been shown that the Calprotectin levels that were initially high had regressed after exclution diet. Thus it is suggested that faecal Calprotectin can help to show fecal inflammation due to CMP, response to exclution diet and followup of the disease activity in CMA. Especially patients in nonIgE mediated group that shows more intestinal signs had higher fecal calprotectin than IgE mediated group that shows less intestinal signs. Thus we can say that non İE mediated group has high intestinal inflammation than non İgE mediated group.

\section{EPIDEMIOLOGY OF INFLAMMATORY BOWEL DISEASE IN CHILDREN - A 2000/2012 COMPARATIVE STUDY}

doi:10.1136/archdischild-2012-302724.0694

${ }^{1} \mathrm{C}$ Becheanu, 'I Tincu, 'R Smadeanu, 'G Lesanu, ${ }^{2} \mathrm{~A}$ Constantinescu, ${ }^{3} \mathrm{~V}$ Hurduc, ${ }^{4} \mathrm{M}$ Munteanu. ${ }^{\prime \prime G r i g o r e ~ A l e x a n d r e s c u '}$ Emergency Children's Hospital; ${ }^{2}$ Clinical Institute 'Fundeni'; ' ${ }^{\prime} \mathrm{Dr}$ Victor Gomoiu' Children's Hospital; " ${ }^{\prime}$ Maria Sklodowska' Emergency Children's Hospital, Bucharest, Romania

Background and Aims Inflammatory bowel disease (IBD) appears to be increasingly common in children even in Eastern Europe. The aim of this paper was to examine the prevalence of children with IBD and to compare the results with a similar study performed twelve years ago.

Methods We report the results of a survey of all the departments of pediatric gastroenterology in Bucharest designed to determine the characteristics of children with IBD. We selected patients with ulcerative colitis (UC) and Cohn's Disease (CD) during a 12 years period (20oo-2012). The patients of the cohort were stratified according to diagnosis $\mathrm{CD}$ and UC, as well as age at first manifestation.

Results There were 21 cases of IBD in children reported in 2000 and 77 children in 2012. From those pediatric patients who were diagnosed as IBD between 2000-2012, we identified 41 cases of UC $(53.2 \%)$ and 36 patients with CD $(46.7 \%)$. The previous study reported 17 (81\%) patients with UC and 4 (19\%) patients with CD. The mean age of onset of symptoms in 2012 was 10.2 years while in 2000 this was 13.2 years. $14.9 \%$ of children are under 5 year of age.

Conclusion Number of patients with IBD has tripled in the last 12 years in our geographical area. UC is still the most frequent form of IBD in children but we report a growing number of CD cases. There is a significant trend for first manifestation occurring at younger ages today compared to the last decade.

\section{EOSINOPHILIC GASTROINTESTINAL DISEASE IN CHILDREN: DIVERSE CLINICAL MANIFESTATIONS}

doi:10.1136/archdischild-2012-302724.0695

${ }^{1} \mathrm{HH}$ Shih, ${ }^{2} \mathrm{~KB}$ Tsai. 'Kaohsiung Municipal Hsiao-Kang Hospital; ' 2 Dept. of Pathology, Kaohsiung Municipal Hsiao-Kang Hospital, Kaohsiung City, Taiwan R.O.C.

Background Clinical manifestations of eosinophilic gastrointestinal diseases (EGIDs) varies a lot in Children. The diagnosis relies on practitian's experience and characteristic pathological features.

Methods A retrospective chart review was conducted of pediatric patients (younger than 18 years old) diagnosed with EGIDs by histopathology proven between 1998 and 2012. Clinical presentations, laboratory data, endoscopic and histopathologic findings were analyzed.

Results Eleven pediatric patients ( 5 boys and 6 girls) were identified and recruited into the study. There were 8 cases (72\%) with eosinophilic gastroenteritis (EGE) and three cases (33\%) with eosinophilic colitis (EoC). The clinical features of EGE included abdominal pain $(6 / 8,75 \%)$, vomiting $(5 / 8,62.5 \%)$, diarrhea $(3 / 8,37.5 \%)$, peripheral eosinophilia $>500$ cells $/ \mathrm{microL}(0 / 6,0 \%)$, elevated $\operatorname{IgE}$ $(4 / 8,50 \%)$, documented specific food IgE (3/7, 43\%). One infant was presented as protein-losing enteropathy. Swollen pyloric ring, edematous duodenal bulb hinted the diagnosis on endoscopy. One patient received appendectomy because of acute abdomen. All patients' acute symptoms got improved after anti-histamine, probiotics, or even short-course steroid. The clinical manifestations of 